\title{
Search for an object with two attributes
}

\author{
Wojciech Olszewski and Asher Wolinsky*
}

May 2015 (First version December 2011)

\begin{abstract}
This paper presents and analyzes a simple equilibrium search model. On one side, there is a population of searchers; on the other side, a population of objects. Searchers are strategic decision makers who are there to get matched to an object, while objects have no preferences or decisions to make. The main novelty is that an object is characterized by two attributes, but a searcher can observe only one at the point of decision. This generates some interesting phenomena such as multiplicity of equilibria, in some of which the searcher's payoff is a discontinuous function of the observed attribute and the adoption decision is not everywhere monotonic in the observed attribute.
\end{abstract}

${ }^{*}$ Olszewski is at the Department of Economics, Northwestern University, Evanston, IL 60208, USA; email: wo@northwestern.edu. Wolinsky is at the Department of Economics, Northwestern University, Evanston, IL 60208, USA, email: a-wolinsky@northwestern.edu. The authors gratefully acknowledge support from the NSF under grants SES-0644930 and SES-1123595, respectively. We are grateful to Keiichi Kawai and Mikhail Safronov for their helpful research assistance, and to Philip H. Dybvig for comments and suggestions.

1

(C) 2015. This manuscript version is made available under the Elsevier user license http://www.elsevier.com/open-access/userlicense/1.0/ 


\section{Introduction}

This paper presents and analyzes a simple equilibrium search model. On one side, a population of identical searchers; on the other side, a population of heterogenous objects. Searchers are strategic decision makers looking for an object. Their preferences over objects are common. Objects have no preferences or decisions to make. The main novelty is that an object is characterized by two attributes, but a searcher can observe only one at the point of decision (which can be viewed as implicitly capturing an underlying cost structure or a time constraint). This generates some interesting phenomena such as multiplicity of equilibria, in some of which the searcher's payoff is a discontinuous function of the information observed and the adoption decision is not everywhere monotonic in that information.

Since our main objective is to expose some possible effects of imperfect information at the decision point in a search environment (rather than to study any particular market), we have opted for a lean model that is not guided by the details of a specific application. Nevertheless, it is useful to observe that the model captures some of the essential features of competitive search for ideas (R\&D).

Other models of search with common values recognize the externalities that searchers exert upon each other through the adverse selection effect of their decisions on the quality of the equilibrium pool (e.g., Burdett and Coles (1997), Davis (2001), Cheron et al. (2011)), or feature searchers who have only partial information at the point of decision (e.g., Chade (2006)), or recognize the endogenous interdependence between the observable duration of search and unobservable worker's quality (e.g., Lockwood (1991)). What distinguishes the present paper from that other work is the combined effect of adverse selection and different searchers observing different attributes. This creates an endogenous interdependence between the attributes, which adds another twist to the duration effect. Here too the expected value of the unobserved attribute is declining in the duration of an object in the process. But the latter is unobservable and enters indirectly through being negatively correlated with the observed attribute. Finally, the idea of search with multi-attributes appears in Neeman (1995) and Bar-Isaac, Caruana and Cunat (2011), but their relation to the present paper is more through the general motivation than through the specific analysis.

\section{Model}

There are two populations of equal size: searchers and objects. Searchers are strategic decision makers looking to get matched to an object; objects have no preferences or decisions to make.

Each object is characterized by two attributes, $\left(x_{1}, x_{2}\right) \in[0,1]^{2}$, whose magnitudes are independent, and differ across objects. A searcher's payoff from adopting an object, characterized by $\left(x_{1}, x_{2}\right)$, is $u\left(x_{1}, x_{2}\right)=$ $x_{1}+x_{2}$.

Upon encountering an object, the searcher observes only one of the attributes.

Time is discrete. In each period, searchers and objects are matched randomly pairwise, so that the maximal number of pairs are formed each period. If a searcher accepts the matched object, both depart; 
otherwise, these searcher and object return to the pools of the unmatched.

A mass $m$ of new searchers and a mass $m$ of new objects enter the market in the beginning of each period. This mass does not depend on the mass of searchers and objects departing the market. Let $H$ denote the CDF of the attributes of the entering objects. The attributes are assumed independent, $H\left(x_{1}, x_{2}\right)=H_{1}\left(x_{1}\right) H_{2}\left(x_{2}\right)$. The marginal distribution $H_{i}$ has full support on $[0,1]$ and is differentiable with density $h_{i}$. Searchers and objects stay in the process until they become a party to a successful match or "die". A fraction $d \in(0,1)$ of all participants die each period. The payoff to a searcher who "dies" before adopting an object is 0 .

A (Markov) strategy for a searcher is a choice of attribute $i \in\{1,2\}$ to observe and an acceptance rule: $A(x) \in\{$ accept,reject $\}$ as a function of the level $x$ of the value of observed attribute $i$.

A particular form of an acceptance rule is a threshold rule $A^{\underline{x}}$ with threshold $\underline{x}$

$$
A^{\underline{x}}(x)=\left\{\begin{array}{l}
\text { accept if } x \geq \underline{x} \\
\text { reject if } x<\underline{x}
\end{array} .\right.
$$

The state of the process at period $t$ is described by masses $M^{t}$ of searchers and of objects, a distribution $F^{t}$ of the attributes of objects present in the process (as distinct from $H$ ) and a distribution of strategies over the searchers' population. These together with $m$ and $H$ determine $M^{t+1}$ and $F^{t+1}$.

This process is in a steady state if $M^{t}, F^{t}$ and the distribution of strategies remain constant over time. Henceforth, attention will be restricted to steady states and the time superscripts will be dropped.

Let $V_{i}(A ; F)$ denote the expected utility of a searcher who observes attribute $i$ and uses the strategy $A$ in a steady state situation with distribution $F$.

An equilibrium is a steady state such that each searcher's strategy $A$ in the support of the distribution of strategies maximizes $V_{i}(A ; F)$ over $i$ and $A$.

\section{The benchmark case}

Suppose that all searchers observe only $x_{1}$, so this is not a matter of choice. Since $E\left(x_{2}\right)$ is independent of $x_{1}$, it is immediate that the searcher's optimal strategy, given $F$, satisfies

$$
A\left(x_{1}\right)=\left\{\begin{array}{l}
\text { accept if } x_{1}+E_{F_{2}}\left(x_{2}\right)>(1-d) V_{1}(A ; F) \\
\text { reject if } x_{1}+E_{F_{2}}\left(x_{2}\right)<(1-d) V_{1}(A ; F)
\end{array} .\right.
$$

That is, $A$ is of the threshold variety $A^{\underline{x}}$ with threshold $\underline{x}=(1-d) V_{1}\left(A^{\underline{x}} ; F\right)-E_{F_{2}}\left(x_{2}\right)$

When all searchers employ the same threshold strategy $A^{\underline{x}}$, the steady state distribution $F^{\underline{x}}\left(x_{1}, x_{2}\right)=$ $F_{1}^{\underline{x}}\left(x_{1}\right) F_{2}^{\underline{x}}\left(x_{2}\right)$ is such that $F_{2}^{\underline{x}}=H_{2}$ and $F_{1}^{\underline{x}}$, has two steps:

$$
F_{1}^{\underline{x}}(x)=\left\{\begin{array}{clc}
\frac{H_{1}(x) / d}{H_{1}(\underline{x}) / d+1-H_{1}(\underline{x})} & \text { if } & x<\underline{x} ; \\
\frac{H_{1}(\underline{x}) / d+H_{1}(x)-H_{1}(\underline{x})}{H_{1}(\underline{x}) / d+1-H_{1}(\underline{x})} & \text { if } & x>\underline{x} .
\end{array}\right.
$$


To understand (3), recall that steady state requires the equality of outflows and inflows of searchers and objects of each type. Since objects with $x_{1}<\underline{x}$ depart only through death, for $x<\underline{x}, d M F_{1}^{\underline{x}}(x)=$ $m H_{1}(x)$, and so $F_{1}^{\underline{x}}(x)=m H_{1}(x) / M d$. Since objects with $x_{1}>\underline{x}$ are accepted immediately, for $x>\underline{x}$, $M\left[F_{1}^{\underline{x}}(x)-F_{1}^{\underline{x}}(\underline{x})\right]=m\left[H_{1}(x)-H_{1}(\underline{x})\right]$, and so $F_{1}^{\underline{x}}(x)=F_{1}^{\underline{x}}(\underline{x})+m\left[H_{1}(x)-H_{1}(\underline{x})\right] / M$. These together with the overall inflow-outflow equality, $m=M\left[F_{1}^{\underline{x}}(\underline{x}) d+1-F_{1}^{\underline{x}}(\underline{x})\right]$, yield (3).

Observe that

$$
V_{1}\left(A^{\underline{x}} ; F\right)=\left[1-F_{1}(\underline{x})\right]\left[E_{F_{1}}\left(x_{1} \mid x_{1}>\underline{x}\right)+E_{F_{2}}\left(x_{2}\right)\right]+F_{1}(\underline{x})(1-d) V_{1}\left(A^{\underline{x}} ; F\right) .
$$

Threshold $\underline{x}^{e}$ is an equilibrium threshold iff $\underline{x}=\underline{x}^{e}$ maximizes $V_{1}\left(A^{\underline{x}} ; F \underline{x}^{e}\right)$.

Since the inflows into the process are constant and all searchers are identical, $V_{1}\left(A^{\underline{x}} ; F^{\underline{x}}\right)$ captures the population payoff average and hence social welfare when all are using threshold $\underline{x}$. Thus, $\underline{x}^{w}$ is the welfare-maximizing threshold iff $\underline{x}=\underline{x}^{w}$ maximizes $V\left(A^{\underline{x}} ; F^{\underline{x}}\right)$.

Proposition 1 (i) For $d<\frac{E_{H_{1}}\left(x_{1}\right)}{E_{H_{1}}\left(x_{1}\right)+E_{H_{2}}\left(x_{2}\right)}, \underline{x}^{e}>0$; for $d \geq \frac{E_{H_{1}}\left(x_{1}\right)}{E_{H_{1}}\left(x_{1}\right)+E_{H_{2}}\left(x_{2}\right)}, \underline{x}^{e}=0$. (ii) For all $d>0$, $x^{w}=0$.

Proof: It follows from (2) that either $\underline{x}^{e}=0$ and $(1-d) V_{1}\left(A^{0} ; F^{0}\right) \leq E_{F_{2}^{0}}\left(x_{2}\right)$ or $\underline{x}^{e} \in(0,1)$ and $\underline{x}_{1}^{e}+E_{F_{2}}\left(x_{2}\right)=(1-d) V_{1}\left(A^{x^{e}} ; F^{x^{e}}\right)$.

$$
\begin{aligned}
V_{1}\left(A^{\underline{x}} ; F^{\underline{x}}\right) & =\left[1-F_{1}^{\underline{x}}(\underline{x})\right]\left[E_{F_{1}^{\underline{x}}}\left(x_{1} \mid x_{1}>\underline{x}\right)+E_{F_{2}^{\underline{x}}}\left(x_{2}\right)\right]+F_{1}^{\underline{x}}(\underline{x})(1-d) V_{1}\left(A^{\underline{x}} ; F^{\underline{x}}\right) \\
& =\left[1-H_{1}(\underline{x})\right]\left[E_{F_{1}^{\underline{x}}}\left(x_{1} \mid x_{1}>\underline{x}\right)+E_{H_{2}}\left(x_{2}\right)\right]=\int_{\underline{x}}^{1} x_{1} d H_{1}\left(x_{1}\right)+\left[1-H_{1}(\underline{x})\right] E_{H_{2}}\left(x_{2}\right) .
\end{aligned}
$$

where the second equality uses (4), $F_{2}^{\underline{x}}=H_{2}$, and $F_{1}^{\underline{x}}(x \mid x>\underline{x})=H_{1}(x \mid x>\underline{x})$. If $d \geq \frac{E_{H_{1}}\left(x_{1}\right)}{E_{H_{1}}\left(x_{1}\right)+E_{H_{2}}\left(x_{2}\right)}$, then (5) implies $(1-d) V_{1}\left(A^{\underline{x}} ; F^{\underline{x}}\right) \leq E_{F_{2}}\left(x_{2}\right)$ for all $\underline{x}$. Since $V_{1}\left(A^{\underline{x}} ; F^{\underline{x}}\right)$, is strictly decreasing in $\underline{x}$, $\underline{x}^{e}=0$ is the unique equilibrium threshold. If $d<\frac{E_{H_{1}}\left(x_{1}\right)}{E_{H_{1}}\left(x_{1}\right)+E_{H_{2}}\left(x_{2}\right)}$, then $\underline{x}+E_{F_{2}}\left(x_{2}\right)=(1-d) V_{1}\left(A^{\underline{x}} ; F^{\underline{x}}\right)$, which by (5) is equivalent to

$$
\underline{x}+E_{H_{2}}\left(x_{2}\right)=(1-d)\left[\int_{\underline{x}}^{1} x_{1} d H_{1}\left(x_{1}\right)+\left[1-H_{1}(\underline{x})\right] E_{H_{2}}\left(x_{2}\right)\right],
$$

has a unique solution $\underline{x}^{e} \in(0,1)$. This is because at $\underline{x}=0, \operatorname{RHS}(6)>\operatorname{LHS}(6)$; at $\underline{x}=1, \operatorname{RHS}(6)<\operatorname{LHS}(6)$; the LHS is increasing in $\underline{x}$ while the RHS is decreasing. This and the inequality at $\underline{x}=0$ imply that the solution $\underline{x}^{e}$ is the unique equilibrium threshold. The observation that RHS(6) is strictly decreasing also implies via (5) that $V_{1}\left(A^{\underline{x}} ; F^{\underline{x}}\right)$ is maximized at $\underline{x}=0$. Hence $x^{w}=0$.

Thus, the equilibrium involves excessive search from social point of view, which is not surprising since searchers do not take into account the adverse effect of their rejection decisions on the pool of objects available to others. 


\section{Two-attribute case}

Suppose now that each searcher chooses one attribute to observe. Consider a steady state in which a fraction $g_{i}$ of the searchers examine attribute $i\left(g_{2}=1-g_{1}\right)$ and employ the same strategy $A_{i}$, and the distribution of attributes present in the process is $F$. If this steady state is an equilibrium, the optimality of the searchers' choice of an attribute to observe implies

$$
\begin{aligned}
& \text { if } \quad g_{i} \in(0,1), \quad \text { then } \quad V_{1}\left(A_{1} ; F\right)=V_{2}\left(A_{2} ; F\right), \\
& \text { if } \quad g_{i}=1, \quad \text { then } \quad V_{i}\left(A_{i} ; F\right) \geq V_{j}\left(A_{j} ; F\right),
\end{aligned}
$$

and the optimality of searchers' acceptance rule implies

$$
\begin{aligned}
& \text { if } A_{i}(x)=\text { accept, then } \quad x+E_{F}\left(x_{j} \mid x_{i}=x\right) \geq(1-d) V_{i}\left(A_{i} ; F\right), \\
& \text { if } A_{i}(x)=\text { reject, then } \quad x+E_{F}\left(x_{j} \mid x_{i}=x\right) \leq(1-d) V_{i}\left(A_{i} ; F\right) .
\end{aligned}
$$

The acceptance behavior partitions $[0,1]^{2}$ into four product sets $R R=\left\{\left(x_{1}, x_{2}\right) \mid A_{1}\left(x_{1}\right)=A_{2}\left(x_{2}\right)=\right.$ reject $\}, A R=\left\{\left(x_{1}, x_{2}\right) \mid A_{1}\left(x_{1}\right)=\right.$ accept; $A_{2}\left(x_{2}\right)=$ reject $\}, R A=\left\{\left(x_{1}, x_{2}\right) \mid A_{1}\left(x_{1}\right)=\right.$ reject; $A_{2}\left(x_{2}\right)=$ accept $\}$, and $A A=\left\{\left(x_{1}, x_{2}\right) \mid A_{1}\left(x_{1}\right)=A_{2}\left(x_{2}\right)=\right.$ accept $\}$. Let $M_{i j}, i j=R R, A R, R A, A A$, denote the masses of objects in these four sets and let $P_{\Psi}(B)$ denote the probability of the set $B \subset[0,1]^{2}$, given a distribution $\Psi$ on $[0,1]^{2}$.

The steady state conditions require the equality of inflows and outflows:

$$
\begin{aligned}
d M_{R R} & =m P_{H}(R R) ;\left[d g_{1}+g_{2}\right] M_{R A}=m P_{H}(R A) ; \\
{\left[g_{1}+d g_{2}\right] M_{A R} } & =m P_{H}(A R) ; M_{A A}=m P_{H}(A A) .
\end{aligned}
$$

That is, objects with attributes in $R R$ exit only through death and hence the outflow is $d M_{R R}$; objects with attributes in $A A$ are adopted immediately and hence the outflow is $M_{A A}$; objects from $R A$ and $A R$ are adopted by searchers who examine one of the attributes, but exit only through death when matched with searchers who examine the other attribute. This determines completely the distribution $F$. Given any set $B \subset[0,1]^{2}$,

$$
P_{F}(B)=\frac{\sum_{i j \in\{R R, A R, R A, A A\}} M_{i j} P_{H}(B \mid i j)}{\sum_{i j \in\{R R, A R, R A, A A\}} M_{i j}}
$$

This implies that the conditional of $F$ on any $k \ell=A A, A R, R A, R R$ coincides with the conditional of $H$ on $k \ell$,

$$
P_{F}(B \mid k \ell)=\frac{P_{F}(B \cap k \ell)}{P_{F}(k \ell)}=\frac{1}{P_{F}(k \ell)} \frac{M_{k \ell} P_{H}(B \cap k \ell \mid k \ell)}{\sum_{i j \in\{R R, A R, R A, A A\}} M_{i j}}=P_{H}(B \mid k \ell),
$$

where the second equality follows from (10), and the third inequality follows from $P_{F}(k \ell)$

$=M_{k \ell} / \sum_{i j \in\{R R, A R, R A, A A\}} M_{i j}$.

Thus, $V_{1}=V_{1}\left(A_{1} ; F\right)$ can be expressed in terms of the masses $M_{i j}$ :

$$
\begin{aligned}
V_{1} & =P_{F}(A A) E_{F}\left(x_{1}+x_{2} \mid\left(x_{1}, x_{2}\right) \in A A\right)+P_{F}(A R) E_{F}\left(x_{1}+x_{2} \mid\left(x_{1}, x_{2}\right) \in A R\right)+P_{F}(R R \cup R A)(1-d) V_{1} \\
& =\frac{M_{A A} E_{H}\left(x_{1}+x_{2} \mid\left(x_{1}, x_{2}\right) \in A A\right)+M_{A R} E_{H}\left(x_{1}+x_{2} \mid\left(x_{1}, x_{2}\right) \in A R\right)+\left(M_{R A}+M_{R R}\right)(1-d) V_{1}}{M_{A A}+M_{A R}+M_{R A}+M_{R R}}
\end{aligned}
$$


The second equality follows from (10) and (11). An analogous expression can be obtained for $V_{2}=$ $V_{2}\left(A_{2} ; F\right)$. By $(9)$, the $M_{i j}$ 's can be expressed in terms of the strategies and the parameters of the model. Therefore the $V_{i}$ 's and the equilibrium conditions (7) and (8) can be expressed in terms of the strategies and the parameters alone, providing the system of inequalities that is necessary and sufficient for strategies to be in equilibrium.

\section{Threshold equilibria and payoff discontinuity}

The simplest equilibrium in which each of the attributes is being examined by some subset of the searchers is of the threshold variety. A fraction of searchers $g_{i} \in(0,1)$ examine attribute $i$ and accept the object iff $x_{i} \geq \underline{x}_{i}$. The sets $R R, R A, A A$ and $A R$ are simple rectangles: $R R=\left[0, \underline{x}_{1}\right) \times\left[0, \underline{x}_{2}\right), R A=\left[0, \underline{x}_{1}\right) \times\left[\underline{x}_{2}, 1\right]$, $A R=\left[\underline{x}_{1}, 1\right] \times\left[0, \underline{x}_{2}\right)$ and $A A=\left[\underline{x}_{1}, 1\right] \times\left[\underline{x}_{2}, 1\right]$, as shown in Figure 1 below. Here too $F$ is the steady state distribution corresponding to this behavior ${ }^{1}$.

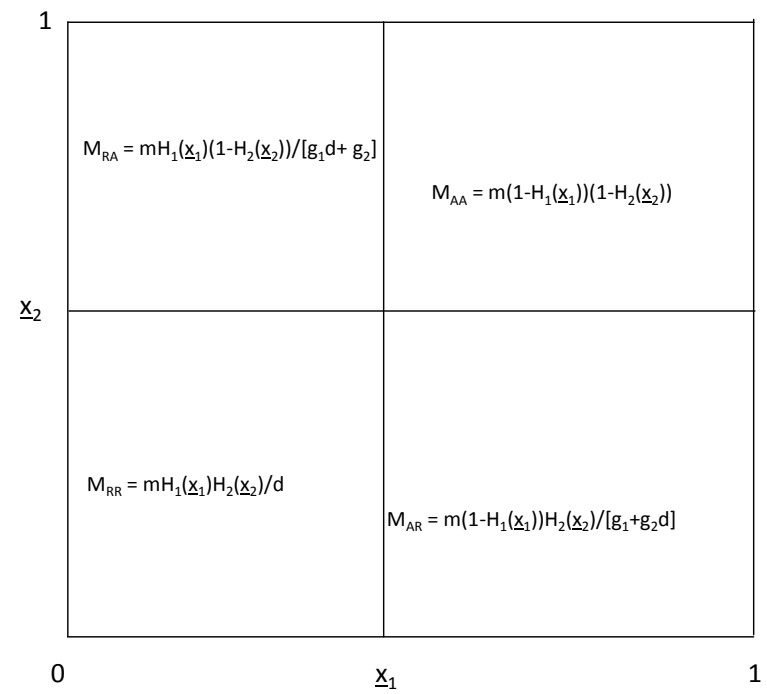

Figure 1: Equilibrium distribution

The key observation for this part of the analysis is the monotonicity of $E_{F}\left(x_{2} \mid x_{1}\right)$ in $x_{1}$ and its discontinuity at $\underline{x}_{1}$ :

Claim $1 E_{F}\left(x_{2} \mid x_{1}\right)$ is constant over $x_{1}<\underline{x}_{1}$ and over $x_{1}>\underline{x}_{1}$, and satisfies

$$
E_{F}\left(x_{2} \mid x_{1}<\underline{x}_{1}\right)<E_{F}\left(x_{2} \mid x_{1}>\underline{x}_{1}\right) .
$$

That $E_{F}\left(x_{2} \mid x_{1}\right)$ is constant over $\left[0, \underline{x}_{1}\right)$ and over $\left(\underline{x}_{1}, 1\right]$ is immediate from the independence of the entering attributes. The inequality in (12) is more intriguing: despite the independence of the entering distributions of attributes, positive correlation emerges in the equilibrium distribution. This translates to discontinuity of the searcher's payoff, $x+E_{F}\left(x_{2} \mid x_{1}=x\right)$, at the threshold.

\footnotetext{
${ }^{1}$ It would be more informative to denote the distribution by $F \underline{x}_{1}, \underline{x}_{2}$ to remind the reader of its dependence on the thresholds, but we forgo this to reduce the density of the notation.
} 
Proof: Observe that (11) implies that $E_{F}\left(x_{2} \mid\left(x_{1}, x_{2}\right) \in k \ell\right)=E_{H_{2}}\left(x_{2} \mid\left(x_{1}, x_{2}\right) \in k \ell\right)$. Also observe that $E_{H_{2}}\left(x_{2} \mid\left(x_{1}, x_{2}\right) \in k R\right)=E_{H_{2}}\left(x_{2} \mid x_{2}<\underline{x}_{2}\right)$ and $E_{H_{2}}\left(x_{2} \mid\left(x_{1}, x_{2}\right) \in k A\right)=E_{H_{2}}\left(x_{2} \mid x_{2}>\underline{x}_{2}\right), k \in\{A, R\}$. Therefore

$$
\begin{array}{ll}
E_{F}\left(x_{2} \quad \mid \quad x_{1}<\underline{x}_{1}\right)=\frac{M_{R R} E_{H_{2}}\left(x_{2} \mid x_{2}<\underline{x}_{2}\right)+M_{R A} E_{H_{2}}\left(x_{2} \mid x_{2}>\underline{x}_{2}\right)}{M_{R A}+M_{R R}} \\
E_{F}\left(x_{2} \quad \mid \quad x_{1}>\underline{x}_{1}\right)=\frac{M_{A R} E_{H_{2}}\left(x_{2} \mid x_{2}<\underline{x}_{2}\right)+M_{A A} E_{H_{2}}\left(x_{2} \mid x_{2}>\underline{x}_{2}\right)}{M_{A R}+M_{A A}} .
\end{array}
$$

Now, (12) follows from

$$
\frac{M_{R A}}{M_{R R}}=\frac{d\left(1-H_{2}\left(\underline{x}_{2}\right)\right)}{\left[d\left(1-g_{2}\right)+g_{2}\right] H_{2}\left(\underline{x}_{2}\right)}<\frac{\left[d\left(1-g_{1}\right)+g_{1}\right]\left(1-H_{2}\left(\underline{x}_{2}\right)\right)}{H_{2}\left(\underline{x}_{2}\right)}=\frac{M_{A A}}{M_{A R}},
$$

that in turn follows from $d<\left[d\left(1-g_{2}\right)+g_{2}\right]\left[d\left(1-g_{1}\right)+g_{1}\right]$ for any $g_{1}, g_{2}>0$ such that $g_{1}+g_{2}=1$. Completely analogous argument establishes the claim for $E_{F}\left(x_{1} \mid x_{2}\right)$.

The explanation is that, on average, objects with $x_{1}<\underline{x}_{1}$ endure for longer in the process than objects with $x_{1}>\underline{x}_{1}$ and are therefore subject to more severe adverse selection with respect to $x_{2}$. That is, if the "age" of an object were observable to a searcher examining attribute 1, an older object would be expected to have a lower $x_{2}$, since it would be more likely to have been encountered and rejected by searchers examining attribute 2 . Now, while the ages of objects are not observable, they are correlated with whether $x_{1}$ is smaller or larger than $\underline{x}_{1}$.

Claim 1 implies immediately that the best response to the threshold configuration is a threshold strategy. Therefore, the equilibrium condition (8) translates to the following condition: if $\underline{x}_{i}$ is an equilibrium threshold, then

$$
\begin{aligned}
& \underline{x}_{i}+E_{F}\left(x_{j} \mid x_{i}>\underline{x}_{i}\right) \geq(1-d) V_{i}\left(A^{\underline{x}_{i}} ; F\right), \text { for } \underline{x}_{i}<1, \\
& \underline{x}_{i}+E_{F}\left(x_{j} \mid x_{i}<\underline{x}_{1}\right) \leq(1-d) V_{i}\left(A^{\underline{x}_{i}} ; F\right), \text { for } \underline{x}_{i}>0 .
\end{aligned}
$$

That is, acceptance of $x_{i}$ just above $\underline{x}_{i}$ yields a higher payoff than the value of continued search, while acceptance of $x_{i}$ just below $\underline{x}_{i}$ yields a lower payoff. Analogous inequalities hold for a searcher who examines attribute 2 .

Consider the symmetric case of $H_{1}=H_{2}$ and a symmetric threshold configuration such that $g_{i}=1 / 2$ and all searchers use the same threshold $\underline{x}$. Let $V^{\underline{x}}=V_{i}\left(A^{\underline{x}} ; F\right)$ and let $v(y, \underline{x})$ denote the expected payoff of a single searcher, who applies threshold $y$ in the first step and continues with the threshold $\underline{x}$ thereafter, that is,

$$
v(y, \underline{x})=P_{F}\left(\left\{\left(x_{1}, x_{2}\right) \mid x_{1} \geq y\right\}\right) E_{F}\left(x_{1}+x_{2} \mid x_{1} \geq y\right)+\left[1-P_{F}\left(\left\{\left(x_{1}, x_{2}\right) \mid x_{1} \geq y\right\}\right)\right](1-d) V^{\underline{x}} .
$$

Thus, $\underline{x}$ is a symmetric equilibrium threshold iff $\underline{x} \in \arg \max _{y} v(y, \underline{x})$. Let $\underline{x}_{\min }$ and $\underline{x}_{\max }$ denote the minimal and maximal symmetric equilibrium thresholds, respectively. Obviously, $\underline{x}_{\max }<1$, since it is suboptimal to reject all objects. 
Proposition 2 Suppose that $H_{1}=H_{2}=H$. A (symmetric) threshold equilibrium exists: (i) for $d<1 / 2$, $\underline{x}_{\min }>0$ and there is a non-degenerate interval $\left[x^{-}, x^{+}\right]$such that every $\underline{x} \in\left[x^{-}, x^{+}\right]$is the threshold of a symmetric threshold equilibrium; (ii) for $d>1 / 2, \underline{x}_{\min }=0$. (iii) If $H$ is uniform, these are all the equilibria: for $d<1 / 2,\left[\underline{x}_{\min }, \underline{x}_{\max }\right]=\left[x^{-}, x^{+}\right]$; for $d>1 / 2, \underline{x}_{\min }=\underline{x}_{\max }=0$.

The proof is in the appendix (as are all subsequent proofs that are not in the body of the paper). Figure 3 describes the uniform case. The diagram for the case of a general $H$ would be similar. Both curves $\left[\underline{x}+E_{F}\left(x_{2} \mid x_{1} \geq \underline{x}\right)\right] /(1-d)$ and $\left[\underline{x}+E_{F}\left(x_{2} \mid x_{1} \leq \underline{x}\right)\right] /(1-d)$ eventually increase as shown in Figure 3. But in the general case, we have not ruled out the possibility that $V \underline{x}$ (the decreasing curve in Figure 3 ) intersects these curves multiple times in the initial range. In such a case, the set of symmetric equilibrium threshold would be a union of intervals.

For equilibrium thresholds in $\left(x^{-}, x^{+}\right)$both inequalities of (14) hold strictly, which means that the value of adopting an object whose observed attribute is just above (below) the threshold is not equal to the value of continued search.

The key to the multiplicity of equilibria is the discontinuity of $x_{1}+E\left(x_{2} \mid x_{1}\right)$ at $x_{1}=\underline{x}$, shown by (12). This produces a kink in $v(y, \underline{x})$ at $y=\underline{x}$. To show this, (9), (10), (13) and (15) are used to obtain an explicit expression for $v(y, \underline{x})$ whose differentiation yields

$$
\operatorname{sgn}\left(\frac{\partial v(y, \underline{x})}{\partial y}\right)=\left\{\begin{array}{lll}
\operatorname{sgn}\left((1-d) V^{\underline{x}}-\left[y+E_{F}\left(x_{2} \mid x_{1}<\underline{x}\right)\right]\right) & \text { if } \quad y<\underline{x} \\
\operatorname{sgn}\left((1-d) V^{\underline{x}}-\left[y+E_{F}\left(x_{2} \mid x_{1}>\underline{x}\right)\right]\right) & \text { if } \quad y>\underline{x}
\end{array} .\right.
$$

The function $v(y, \underline{x})$ is continuous and single-peaked as a function of $y$. If $\underline{x}$ is an equilibrium threshold, $\frac{\partial v(y, \underline{x})}{\partial y} \geq 0$ for $y<\underline{x}$ and $\frac{\partial v(y, \underline{x})}{\partial y} \leq 0$ for $y>\underline{x}$. When these inequalities hold strictly at some $\underline{x}$, then they are satisfied at nearby thresholds as well. Therefore, such $\underline{x}$ belongs to an interval of equilibrium thresholds.

The discontinuity of $E_{F}\left(x_{i} \mid x_{j}\right)$ at $\underline{x}_{j}$ and its implications are not artefacts of the symmetry. Similar discontinuities and multiplicity would also arise in the case of asymmetrically distributed attributes and asymmetric equilibria. Figure 4 in Appendix B depicts the sets of equilibrium thresholds in asymmetric equilibria for uniform $H_{i}$ 's. The multiplicity of threshold equilibria is also robust to some heterogeneity in the preferences. If the extent of the heterogeneity is small relative to the magnitude of the jump of $E_{F}\left(x_{2} \mid x_{1}=\underline{x}\right)$ at $\underline{x} \in\left(x^{-}, x^{+}\right)$, then the symmetric equilibrium with threshold $\underline{x}$ of the original model would still be an equilibrium of the perturbed model. By the same logic, even with more significant heterogeneity, there could ${ }^{2}$ be an equilibrium in which a certain mass of searchers use threshold $\underline{x}$.

In contrast, in the benchmark case of Section 3 in which all searchers examine only attribute $1, x_{1}+$ $E\left(x_{2} \mid x_{1}\right)$ is continuous at the equilibrium threshold, $\underline{x}^{e}$, and the payoff of adopting an object whose observed attribute is $\underline{x}^{e}$ coincides with the value of continued search. Also $\underline{x}^{e}$ is smaller than all symmetric equilibrium thresholds of the two-attribute case.

\footnotetext{
${ }^{2}$ While the intuition is fairly obvious, we have not analyzed this variation formally.
} 


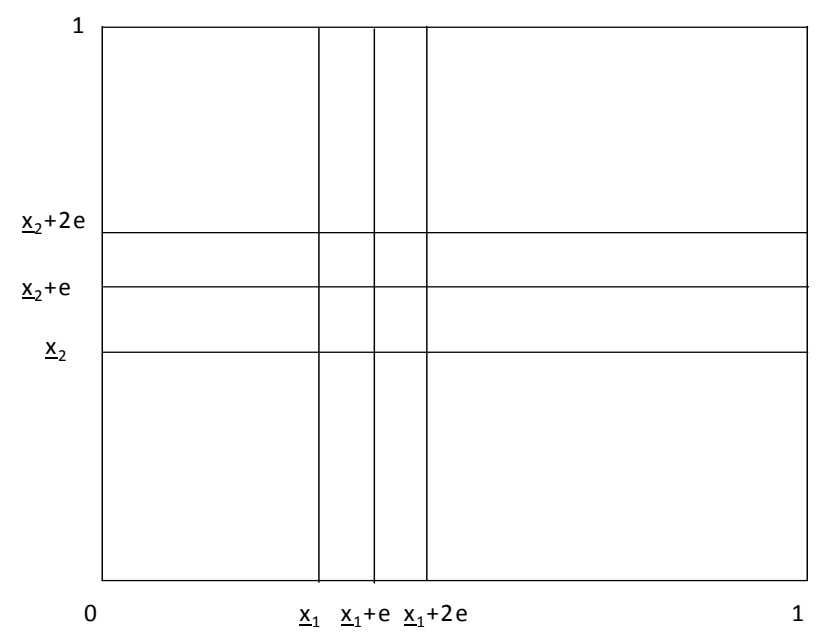

Figure 1: Figure 2: Non-monotonic equilibrium distribution

Proposition 3 Suppose that $H_{1}=H_{2}=H$. Then $\underline{x}^{e} \leq \underline{x}_{\min }$, with strict inequality if $d<1 / 2$.

\section{Non-monotonicity}

Non-monotonic behavior is another implication of the discontinuity of $E_{F}\left(x_{i} \mid x_{j}\right)$ at $\underline{x}_{j}$. Consider a symmetric threshold equilibrium, with threshold $\underline{x}$, for which inequalities (14) hold strictly and modify the acceptance rule as follows

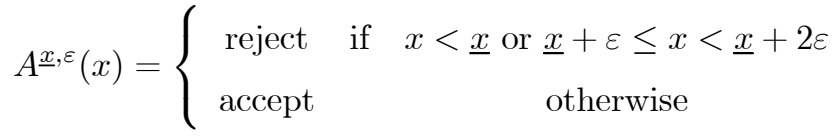

Figure 2 depicts the partition of $[0,1]^{2}$ induced by this acceptance rule. In this configuration $E\left[x_{2} \mid\right.$ $\left.x_{1}<\underline{x}\right]=E\left[x_{2} \mid x_{1} \in[\underline{x}+\varepsilon, \underline{x}+2 \varepsilon)\right]$ and $E\left[x_{2} \mid x_{1} \geq \underline{x}+2 \varepsilon\right]=E\left[x_{2} \mid x_{1} \in[\underline{x}, \underline{x}+\varepsilon)\right]$. It follows that, if the width of these strips, $\varepsilon$, is small enough, the symmetric configuration in which all searchers employ strategy $A^{\underline{x}, \varepsilon}$ is an equilibrium. Let $F^{\underline{x}}, \varepsilon$ denote the steady state distribution compatible with this behavior. First, by symmetry, $V_{1}\left(A^{\underline{x}, \varepsilon} ; F^{\underline{x}, \varepsilon}\right)=V_{2}\left(A^{\underline{x}, \varepsilon} ; F^{\underline{x}, \varepsilon}\right)$. Second, since the inequalities in (14) hold strictly in the original equilibrium, their appropriate versions for this case continue to hold: $V_{i}\left(A^{\underline{x}, \varepsilon} ; F^{\underline{x}, \varepsilon}\right)$, $E_{F \underline{x}, \varepsilon}\left[x_{2} \mid x_{1}<\underline{x}\right]$ and $E_{F \underline{x}, \varepsilon}\left[x_{2} \mid x_{1} \geq \underline{x}+2 \varepsilon\right]$ are " $\varepsilon$-close" to the values of $V_{i}\left(A^{\underline{x}} ; F \underline{\underline{x}}\right), E_{F \underline{x}}\left[x_{2} \mid x_{1}<\underline{x}\right]$ and $E_{F \underline{x}}\left[x_{2} \mid x_{1} \geq \underline{x}\right]$, respectively, in the original equilibrium.

The above observations prove the following proposition.

Proposition 4 For $d<1 / 2$, there also exist equilibria that exhibit non-monotonic behavior. Specifically, let $\left[x^{-}, x^{+}\right]$be an interval of symmetric equilibrium thresholds. For any $z \in\left(x^{-}, x^{+}\right)$, there exists an 
equilibrium in which some $x<z$ is accepted and some $x>z$ is rejected. ${ }^{3}$

Observe that in the same way we constructed a non-monotonic equilibrium, we can construct equilibria with an arbitrary finite number of alternating acceptance and rejection intervals. Moreover, the acceptance and rejection regions may be fairly exotic sets rather than just unions of intervals. Simply consider a symmetric equilibrium for which (14) hold with strict inequalities and modify the acceptance rule to

$$
A(x)=\left\{\begin{array}{cc}
\text { reject } & \text { if } \\
\text { accept } & \text { otherwise }
\end{array}\right.
$$

where $C$ is a positive-measure subset of interval $[\underline{x}+\varepsilon, \underline{x}+2 \varepsilon)$. Finally, the existence of non-monotone equilibria is robust to some heterogeneity in the preferences, for the same reason that the payoff discontinuity at the threshold is robust (as pointed out in Section 5).

\section{Diversified and concentrated search}

In the equilibria discussed in the previous sections, the searchers spread themselves across the attributes. This section inquires whether this diversification is a necessary consequence of the interaction. Intuitively, the answer is not obvious. If all are checking attribute 1, its steady state distribution will be worse, implying that it is more prudent to check it. But, for the same reason, it is easier to find an object with a high level of attribute 2 . In general, both patterns might arise in equilibrium.

The next proposition points out a class of distributions of arriving objects for which the latter consideration prevails and, in any equilibrium, each attribute is examined by a strict subset of the searchers. Consider the class of distributions $H$ on $[0,1]$ that satisfy

$$
E_{H}(z \mid z<x)+E_{H}(z \mid z \geq x) \geq x+E_{H}(x) \text {, for every } x
$$

which includes $H(x)=x$ and $H(x)=\sqrt{x}$.

Proposition 5 If $H_{1}=H_{2}=H$ satisfy (17), then there is no equilibrium in which all searchers examine the same attribute.

Proof: Suppose that in some equilibrium all searchers examine attribute 1. It follows from Section 3 that the common acceptance rule is $A^{\underline{x}}$ with some threshold $\underline{x}, F_{2}^{\underline{x}}=H_{2}, F_{1}^{\underline{x}}$ is given by (3) and

$$
(1-d) V_{1}^{\underline{x}}=\underline{x}+E_{F_{2}^{\underline{x}}}\left(x_{2}\right)
$$

Consider the following searcher's strategy: check attribute 2 once; accept the object if $x_{2} \geq y$; otherwise continue with the equilibrium strategy. Let $v(y, \underline{x})$ denote the a searcher's expected utility of this strategy

\footnotetext{
${ }^{3}$ The range over which this proposition holds might extend beyond the interval $\left[x^{-}, x^{+}\right]$, but its exact characterization might not contribue a great deal to our discussion.
} 
in the equilibrium considered here

$$
v(y, \underline{x})=(1-H(y))\left[E_{F_{1}}\left(x_{1}\right)+E_{H}\left(x_{2} \mid x_{2} \geq y\right)\right]+H(y)(1-d) V_{1}^{\underline{x}}
$$

is the expected payoff of a single searcher who deviates to this strategy. If $y=\underline{x}$, the outcome of this deviation differs from that of the equilibrium behavior only when the first sampled object is such that $x_{1}<\underline{x}$ and $x_{2} \geq \underline{x}$ or such that $x_{1} \geq \underline{x}$ and $x_{2}<\underline{x}$. In the former case the object is rejected under the equilibrium strategy (yielding continuation payoff $(1-d) V_{1}^{\underline{x}}$ ) but is accepted under the deviation, while in the latter case the reverse is true. Since

$$
E_{F \underline{x}}\left(x_{1}+x_{2} \mid x_{1}<\underline{x}, x_{2} \geq \underline{x}\right)=E_{H}\left(x_{1} \mid x_{1}<\underline{x}\right)+E_{H}\left(x_{2} \mid x_{2} \geq \underline{x}\right)=E_{F \underline{x}}\left(x_{1}+x_{2} \mid x_{1} \geq \underline{x}, x_{2}<\underline{x}\right),
$$

while $P_{F \underline{x}}\left(x_{1}<\underline{x}, x_{2} \geq \underline{x}\right)>P_{F \underline{x}}\left(x_{1} \geq \underline{x}, x_{2}<\underline{x}\right)$, it follows that, if $E_{H}\left(x_{1} \mid x_{1}<\underline{x}\right)+E_{H}\left(x_{2} \mid x_{2} \geq \underline{x}\right) \geq$ $(1-d) V_{1}^{\underline{x}}$, then $v(\underline{x}, \underline{x}) \geq V_{1}^{\underline{x}} \cdot{ }^{4}$ This together with (18) implies that $v(\underline{x}, \underline{x}) \geq V_{1}^{\underline{x}}$ for any $H$ satisfying (17). Now

$$
\left(\frac{\partial v(y, \underline{x})}{\partial y}\right)_{y=\underline{x}}=h(\underline{x})\left[(1-d) V_{1}^{\underline{x}}-E_{F_{1}^{\underline{x}}}\left(x_{1}\right)-\underline{x}\right]>0
$$

where the inequality follows from (18) and $E_{H}\left(x_{2}\right)=E_{F_{2}}\left(x_{2}\right)>E_{F_{1}^{\underline{x}}}\left(x_{1}\right)$. Hence, $v(y, \underline{x})>V_{1}^{\underline{x}}$, for some $y>\underline{x}$, contradicting the supposition that there exists an equilibrium in which all examine attribute 1 .

But equilibrium need not always exhibit diversified search. The following example shows that it is possible to have an equilibrium in which all searchers check the same attribute. Furthermore, for some parameter values such an equilibrium coexists with a diversified equilibrium in which each of the attributes is checked by a subset of searchers.

Suppose that each attribute has two potential values, $x_{i} \in\{0,1\}$, and let $p_{i}=\operatorname{Pr}\left(x_{i}=1\right) \in(0,1)$ at the entry stage. ${ }^{5}$ If $d$ is large, there will be an equilibrium in which searchers adopt the first object they encounter. We will focus on the case of sufficiently small $d$ that induces searchers to exhibit selective behavior of rejecting an object if the examined attribute has value 0 . The term selective equilibrium will be used to describe equilibria exhibiting this behavior.

Proposition 6 Consider the symmetric case, $p_{1}=p_{2}=p$. (i) There is $d^{*}>0$ such that, for any $d<d^{*}$, there exists a symmetric selective equilibrium in which each of the attributes is examined by a half of searchers. (ii) If $p(1+p)>1$, there is $d^{* *}>0$ such that, for any $d<d^{* *}$, there also exists an equilibrium in which all searchers examine attribute 1.

That is, for small enough $d$ 's and large enough $p$ 's, symmetric selective equilibria coexist with equilibria in which all searchers check the same attribute.

\footnotetext{
${ }^{4}$ Notice that $v(\underline{x}, \underline{x})$ is obtained from $V_{1}^{\underline{x}}$ by replacing $(1-d) V_{1}^{\underline{x}}$ with $E_{H}\left(x_{1} \mid x_{1}<\underline{x}\right)+E_{H}\left(x_{2} \mid x_{2} \geq \underline{x}\right)$ on $\left\{\left(x_{1}, x_{2}\right) \mid x_{1}<\underline{x}\right.$, $\left.x_{2} \geq \underline{x}\right\}$, and replacing $E_{H}\left(x_{1} \mid x_{1} \geq \underline{x}\right)+E_{H}\left(x_{2} \mid x_{2}<\underline{x}\right)=E_{H}\left(x_{1} \mid x_{1}<\underline{x}\right)+E_{H}\left(x_{2} \mid x_{2} \geq \underline{x}\right)$ with $(1-d) V_{1}^{\underline{x}}$ on $\left\{\left(x_{1}, x_{2}\right) \mid x_{1} \geq\right.$ $\left.\underline{x}, x_{2}<\underline{x}\right\}$.

${ }^{5}$ Notice that we depart here from the assumption that $H$ is a continuous.
} 


\section{Welfare}

Recall that the equilibrium of the benchmark scenario exhibits excessive search relative to the no search scenario that maximizes searchers' expected utility at the time of entering the market. This is also true in the two-attribute scenario for the same reason. In addition, if one symmetric threshold equilibrium has lower thresholds than another, it would yield higher welfare.

The welfare comparison between the benchmark case and the symmetric threshold equilibrium of the two attribute case is affected by two opposing considerations. If the equilibrium thresholds of both of these scenarios were the same, the benchmark scenario would involve more failed $\operatorname{search}^{6}$ and hence a lower equilibrium payoff. But by Proposition 3, the threshold in the benchmark case is lower, which works to increase the equilibrium payoff. In the uniform case, the former consideration overshadows the latter.

Proposition 7 Suppose that the $H_{i}$ 's are uniform. Any symmetric threshold equilibrium yields higher payoff than the unique equilibrium of the benchmark scenario.

Intuitively, this result seems to have a broader validity, for example, one can easily check that it also holds in the discrete example of Section (7). But we have no characterization of the class of $H$ 's for which it holds.

How does non-monotonicity affect the payoff? Obviously, a symmetric threshold configuration with the same total acceptance probability would yield a higher payoff, since it differs only in that it rejects inferior objects. But, the question is whether a threshold configuration with the same acceptance probability can be sustained as an equilibrium. In the uniform case the answer is affirmative.

Proposition 8 Suppose that the $H_{i}$ 's are uniform. Any symmetric non-monotonic equilibrium yields a lower payoff than some symmetric threshold equilibrium.

This result too seems to have a broader validity, but we have no characterization of how general it is.

\section{$9 \quad$ References}

Bar-Isaac, H., Caruana, G. and V. Cuñat (2011), "Information-Gathering Externalities for a MultiAttribute Good," Journal of Industrial Economics, 60, 162 - 185.

Burdett, K. and M. Coles (1997), "Marriage and Class," Quarterly Journal of Economics, 112, 141 168.

Chade, H. (2006), "Matching with Noise and the Acceptance Curse," Journal of Economic Theory, $129,81-113$.

\footnotetext{
${ }^{6}$ See the proof of Proposition 3.
} 
Chéron, A. Harault, J.-O. and F. Langot (2011), "Age-Dependent Employment Protection," Economic Journal, 121, 1477-1504.

Davis, S. (2001), "The Quality Distribution of Jobs and the Structure of Wages in Search Equilibrium," WP 8434, NBER.

Lockwood, B. (1991), "Information Externalities in the Labour Market and the Duration of Unemployment," Review of Economic Studies, 58, 733-753.

Neeman, Z. (1995), "On Determining the Importance of Attributes with a Stopping Problem," Mathematical Social Science, 29, 195 - 212.

\section{Appendix A}

Proof of Proposition 2: For the case $H_{1}=H_{2}=H$, (14) implies: There exists a symmetric equilibrium with threshold $\underline{x}$ iff

$$
(1-d) V^{\underline{x}}-E_{F}\left(x_{2} \mid x_{1}>\underline{x}\right) \leq \underline{x} \leq(1-d) V \underline{x}-E_{F}\left(x_{2} \mid x_{1}<\underline{x}\right) \text { for } \underline{x}>0,
$$

only the left inequality must hold for $\underline{x}=0$. (There clearly exists no equilibrium for $\underline{x}=1$, because the right inequality does not hold.)

Observe that $V \underline{x}$ is decreasing in $\underline{x}$ and continuous, $V^{0}=2 E_{H}(x)$ and $V^{1}=0$. Also, observe that $\underline{x}+$ $E_{F}\left(x_{2} \mid x_{1}>\underline{x}\right)$ and $\underline{x}+E_{F}\left(x_{2} \mid x_{1}<\underline{x}\right)$ are continuous in $\underline{x}, E_{F}\left(x_{2} \mid x_{1} \geq 0\right)=E_{F}\left(x_{2} \mid x_{1} \leq 0\right)=E_{H}\left(x_{2}\right)=$ $E_{F}\left(x_{2} \mid x_{1} \geq 1\right)=E_{F}\left(x_{2} \mid x_{1} \leq 1\right)\left(\right.$ where $E_{F}\left(x_{2} \mid x_{1} \leq 0\right)=\lim _{\underline{x} \rightarrow 0} E_{F}\left(x_{2} \mid x_{1} \leq \underline{x}\right)$ and $E_{F}\left(x_{2} \mid x_{1} \geq 1\right)=$ $\left.\lim _{\underline{x} \rightarrow 1} E_{F}\left(x_{2} \mid x_{1} \geq \underline{x}\right)\right)$.

Therefore, if $d>1 / 2$, then $\underline{x}=0$ satisfies the left inequality in (19).

If $d<1 / 2$, then $\underline{x}+E_{F}\left(x_{2} \mid x_{1} \geq \underline{x}\right)$ and $\underline{x}+E_{F}\left(x_{2} \mid x_{1} \leq \underline{x}\right)$ are below $(1-d) V \underline{x}$ for $\underline{x}=0$ and above it for $\underline{x}=1$. Therefore, each of the two inequalities in (19) holds with equality for some $\underline{x} \in(0,1)$. Let $\underline{x}_{\min }$ and $\underline{x}_{\max }$ be the smallest and largest such $\underline{x}^{\prime}$ 's respectively. By (12), the left inequality of (19) holds with equality at $\underline{x}_{\min }$, the right inequality holds with equality at $\underline{x}_{\max }$ and $\underline{x}_{\min }<\underline{x}_{\max }$. In addition, there must be some $\underline{x} \in\left[\underline{x}_{\min }, \underline{x}_{\max }\right]$ such that the left inequality of (19) holds with equality at $\underline{x}$ and with strict inequality at all $x>\underline{x}$. Let $x^{-}$denote the minimal $\underline{x}$ with this property. It follows from (12 that the right inequality in (19) holds at $x^{-}$strictly. Let $x^{+}$denote the minimal $\underline{x}>x^{-}$at which the right inequality in (19) holds with equality. Obviously, such $x^{+}$exists and $x^{+} \leq \underline{x}_{\max }$. By construction, (19) holds for any $\underline{x} \in\left[x^{-}, x^{+}\right]$, which therefore is the threshold of a symmetric equilibrium.

For uniform $H, V^{\underline{x}}=(1-\underline{x})^{2}(1+\underline{x})+\frac{1}{d+1} \underline{x}(1-\underline{x})(2 \underline{x}+1), E_{F}\left(x_{2} \mid x_{1}>\underline{x}\right)=\frac{\underline{x}}{2}+\frac{(1+d)(1-\underline{x})}{2(1+d)(1-\underline{x})+4 \underline{x}}$ and $E_{F}\left(x_{2} \mid x_{1}<\underline{x}\right)=\frac{x}{2}+\frac{d(1-\underline{x})}{(1+d) \underline{x}+2 d(1-\underline{x})}$. For $d>1 / 2, V \underline{x}$ is above $\underline{x}+E_{F}\left(x_{2} \mid x_{1}<\underline{x}\right)$ and $\underline{x}+E_{F}\left(x_{2} \mid\right.$ $x_{1}>\underline{x}$ ) for all $\underline{x}$. The case of $d<1 / 2$ is shown in Figure 3. $V \underline{x}$ intersects with $\underline{x}+E_{F}\left(x_{2} \mid x_{1}<\underline{x}\right)$ and $\underline{x}+E_{F}\left(x_{2} \mid x_{1}>\underline{x}\right)$ in their upward sloping part (in fact, the latter curve is upward-sloping throughout the range) and therefore only once with each. 
Proof of Proposition 3: For $d \geq 1 / 2$, the result is immediate. Let $\hat{V}_{1}^{\underline{x}}$ denote the expected payoff of search with threshold $\underline{x}$ in the benchmark case in which all examine attribute 1 . First, observe that, for all $\underline{x}, \hat{V}_{1}^{\underline{x}}<V^{\underline{x}}$. This can be seen from the perspective of each entering cohort of the arriving objects. In both scenarios, objects with $x_{1}>\underline{x}$ and $x_{2}>\underline{x}$ are always picked up and objects with $x_{1}<\underline{x}$ and $x_{2}<\underline{x}$ are never picked up. So the difference is with respect to objects with one attribute above and the other below $\underline{x}$. In the benchmark scenario, half of these objects are picked up (those with $x_{1}>\underline{x}$ ) and the other half are never picked up. In the symmetric scenario a fraction of

$$
1 / 2+(1-d)(1 / 4)+(1-d)^{2}(1 / 8)+\ldots=1 /(1+d)>1 / 2
$$

of these objects are picked up. Therefore, the payoff extracted from each entering cohort of objects, and hence the overall payoff is higher in the latter scenario. The equilibrium threshold in the benchmark case is the solution to $\underline{x}+E_{H}\left(x_{2}\right)=(1-d) \hat{V}_{1}^{\underline{x}}$, while $\underline{x}_{\min }$ in the symmetric scenario is the solution to $\underline{x}+E_{F}\left(x_{2} \mid x_{1}>\underline{x}\right)=(1-d) V \underline{x}$. Therefore, $\hat{V}_{1}^{\underline{x}}<V \underline{x}$ and $E_{H}\left(x_{2}\right)>E_{F}\left(x_{2} \mid x_{1}>\underline{x}\right)$, for all $\underline{x}$, imply the result.

Proof of Proposition 6:The steady state equilibrium distribution $F$ is described by the fractions $f_{j \ell}=$ $\operatorname{Pr}\left(x_{1}=j, x_{2}=\ell\right), j, \ell=0,1$. Since the equilibrium acceptance policy always has $A_{i}(1)=$ accept, we may write $V_{i}(A ; F)$ as $V_{i}\left(A_{i}(0) ; F\right)$.

$$
V_{1}\left(A_{i}(0) ; F\right)=\left\{\begin{array}{ccc}
\left(f_{10}+f_{11}\right)\left(1+\frac{f_{11}}{f_{10}+f_{11}}\right)+\left(f_{00}+f_{01}\right)(1-d) V_{i}\left(A_{1}(0) ; F\right) & \text { if } & A_{1}(0)=\text { reject } \\
\left(f_{10}+f_{11}\right)\left(1+\frac{f_{11}}{f_{10}+f_{11}}\right)+\left(f_{00}+f_{01}\right) \frac{f_{01}}{f_{00}+f_{01}} & \text { if } & A_{1}(0)=\text { accept }
\end{array},\right.
$$

which yields

$$
V_{1}(\text { reject } ; F)=\frac{f_{10}+2 f_{11}}{f_{10}+f_{11}+d\left(f_{00}+f_{01}\right)} \text { and } V_{1}(\text { accept } F)=1+f_{11}-f_{00} ;
$$

$V_{2}($ reject $F)$ is derived analogously (the roles of 01 and 10 are exchanged); and $V_{2}$ (accept; $\left.F\right)=V_{1}($ accept; $F)$.

When searchers exhibit selective behavior, $f_{j \ell}$ can be expressed in terms of the parameters as follows. Let $M_{j \ell}, j, \ell \in\{0,1\}$, denote the mass of objects whose attributes are $j$ and $\ell$. Recall that $g_{i}$ is the population fraction examining attribute $i$, and $m$ is the mass of searchers and of objects entering each period. By the steady state equality of inflows and outflows,

$$
\begin{aligned}
M_{11} & =m p_{1} p_{2}, d M_{00}=m\left(1-p_{1}\right)\left(1-p_{2}\right) \\
M_{10} g_{1}+M_{10} g_{2} d & =m p_{1}\left(1-p_{2}\right), M_{01} g_{2}+M_{01} g_{1} d=m\left(1-p_{1}\right) p_{2} .
\end{aligned}
$$

Therefore,

$$
f_{10}=\frac{M_{10}}{M_{00}+M_{10}+M_{01}+M_{11}}=\frac{\frac{p_{1}\left(1-p_{2}\right)}{g_{1}+g_{2} d}}{\frac{\left(1-p_{1}\right)\left(1-p_{2}\right)}{d}+\frac{p_{1}\left(1-p_{2}\right)}{g_{1}+g_{2} d}+\frac{\left(1-p_{1}\right) p_{2}}{g_{2}+g_{1} d}+p_{1} p_{2}},
$$

and $f_{00}, f_{01}$ and $f_{00}$ are calculated similarly from $(22)$. 
By plugging (23) and the analogous expressions for the other $f_{i j}$ 's into $(21), V_{i}\left(A_{i}(0) ; F\right)$ can be expressed in terms of the parameters $p_{i}$ and $d$, and the variable $g_{i}$. In the symmetric case $p_{1}=p_{2}=p$ and for the symmetric configuration, we have that $g_{1}=g_{2}=1 / 2$, and

$$
V_{i}(\text { reject } F) \geq V_{j}(\text { reject } F) \text { and }(1-d) V_{i}(\text { reject } F) \geq V_{j}(\text { accept } F) \text {. }
$$

The first of these inequalities is satisfied by symmetry. Therefore, a symmetric selective equilibrium exists iff

$$
(1-d) V_{i}(\text { reject } F)=(1-d) 2 p \frac{1+d p}{1+d} \geq d 2 p \frac{1+(1-p+p d)}{d+(1-p+p d)^{2}}=V_{j}(\text { accept } F) .
$$

Since the RHS goes to 0 with $d$ going to 0 , while the LHS is bounded away from 0 , for sufficiently small $d$, this inequality holds and hence such a symmetric equilibrium exists.

Still in the case of $p_{1}=p_{2}=p$, there exists a selective equilibrium in which all searchers check attribute $1\left(g_{1}=1, g_{2}=0\right)$, iff

$$
(1-d) V_{1}(\text { reject; } F)=(1-d) p(1+p) \geq p \frac{d+p d+1-p}{1-p+d p}=V_{1}(\operatorname{accept} ; F),
$$

and

$$
V_{1}(\text { reject } ; F)=p(1+p) \geq \frac{\frac{p(1-p)}{d}+2 p^{2}}{p(1-p) \frac{(1-d)^{2}}{d}+1}=V_{2}(\text { reject } F) .
$$

The first these conditions holds iff $d^{2} p(1+p)+2 d(1-p)(1+p)-p(1-p) \leq 0$, and the second condition holds iff $p(1+p)(1-d) \geq 1$. Since $p \in(0,1)$, the first condition is satisfied for small enough $d$. And if $p(1+p)>1$, the second condition is also satisfied for small enough $d$

Proof of Proposition 7: The lowest payoff among all symmetric threshold equilibrium is attained with $\underline{x}=x^{+}$(see Proposition 2). From the proof of Proposition 2, $x^{+}$and $V^{x^{+}}$are jointly determined (for uniform $H$ ) by

$V^{x^{+}}=\left(1-x^{+}\right)^{2}\left(1+x^{+}\right)+\frac{1}{d+1} x^{+}\left(1-x^{+}\right)\left(2 x^{+}+1\right)$ and $\frac{3 x^{+}}{2}+\frac{d\left(1-x^{+}\right)}{(1+d) x^{+}+2 d\left(1-x^{+}\right)}=(1-d) V^{x^{+}}$.

By (6) and (5), the unique equilibrium threshold $\underline{x}^{e}$ and payoff $V_{1}^{\underline{x}^{e}}$ of the benchmark scenario in which all examine attribute 1 (and $H$ is uniform) satisfy

$$
V_{1}^{\underline{x}^{e}}=\frac{\left(1-\underline{x}^{e}\right)\left(2+\underline{x}^{e}\right)}{2} \text { and } \underline{x}^{e}+\frac{1}{2}=(1-d) V_{1}^{\underline{x}^{e}} .
$$

By solving directly the equations, we obtain $V^{x^{+}}>V_{1}^{x^{e}}$ for any $d \in(0,1 / 2)$ (See Figure 5 in Appendix B).

Proof of Proposition 8: Let superscript $n m$ index magnitudes corresponding to the symmetric nonmonotonic equilibrium. Let $A^{n m}=A_{1}^{n m}=A_{2}^{n m}$ be the equilibrium acceptance rule and let

$$
\underline{x}=\text { measure of }\left\{x: A^{n m}(x)=\text { reject }\right\} .
$$


Observe that $\underline{x}=y+z$, where

$$
y=\inf \left\{x: A^{n m}(x)=\operatorname{accept}\right\} \text { and } z=\text { measure of }\left\{x \geq y: A^{n m}(x)=\text { reject }\right\} .
$$

A threshold equilibrium with lower threshold than $\underline{x}$ would obviously yield higher payoff than this nonmonotonic equilibrium. So, the non-monotonic equilibrium can yield higher welfare only when $\underline{x}<x^{-}$. Consider this case.

By the definition of $y$,

$$
y+E^{n m}\left(x_{2} \mid x_{1} \text { s.t. } A^{n m}\left(x_{1}\right)=\text { accept }\right) \geq(1-d) V^{n m},
$$

Since $\underline{x}+E\left(x_{2} \mid x_{1}\right.$ s.t. $\left.x_{1}>\underline{x}\right)$ is increasing in $\underline{x}$ (see Figure 3 at the end of this appendix), and $x^{-}+E\left(x_{2} \mid x_{1}\right.$ s.t. $\left.x_{1}>x^{-}\right)=(1-d) V^{x^{-}}$by the definition of $x^{-}$, we have

$$
\underline{x}+E\left(x_{2} \mid x_{1} \text { s.t. } x_{1}>\underline{x}\right) \leq(1-d) V^{x^{-}} .
$$

(Recall that we have assumed that $\underline{x}<x^{-}$.) It follows from (25) and (26) that in order to establish that $V^{n m} \leq V^{x^{-}}$, it suffices to show that

$$
E^{n m}\left(x_{2} \mid x_{1} \text { s.t. } A^{n m}\left(x_{1}\right)=\text { accept }\right)-E\left(x_{2} \mid x_{1} \text { s.t. } x_{1}>\underline{x}\right) \leq z .
$$

Consider a symmetric non-monotonic configuration in which an object is accepted iff the examined attribute belongs to $[y, 1-z]$. Let $E^{[y, 1-z]}\left(x_{2} \mid x_{1}\right.$ s.t. $\left.y \leq x_{1} \leq 1-z\right)$ denote the expectation corresponding to that configuration. Observe that $E^{[y, 1-z]}\left(x_{2} \mid x_{1}\right.$ s.t. $\left.y \leq x_{1} \leq 1-z\right) \geq E^{n m}\left(x_{2} \mid x_{1}\right.$ s.t. $A^{n m}\left(x_{1}\right)=$ accept). This is because, like in the $n m$-configuration, the rejection region of the $[y, 1-z]-$ configuration consists of $[0, y]$ and a region of measure $z$. But in the $[y, 1-z]$-configuration, the rejection region of measure $z$ is located at the upper end, which means that the distribution of the unobserved attribute stochastically dominates the corresponding distribution in the $n m$-configuration. Therefore, it suffices to show that

$$
E^{[y, 1-z]}\left(x_{2} \mid x_{1} \text { s.t. } y \leq x_{1} \leq 1-z\right)-E\left(x_{2} \mid x_{1} \text { s.t. } x_{1}>\underline{x}\right) \leq z .
$$

Direct calculations show that indeed

$$
E^{[y, 1-z]}\left(x_{2} \mid x_{1} \text { s.t. } y \leq x_{1} \leq 1-z\right)-E\left(x_{2} \mid x_{1} \text { s.t. } x_{1}>\underline{x}\right)=z \frac{\frac{1-d}{1+d}(1-y-z)}{\left(\frac{2}{d+1}\right)(y+z)+(1-y-z)} \leq z,
$$

where the last inequality follows from $0 \leq y+z \leq 1$.

Figure 3 illustrates how $x^{-}$and $x^{+}$(from the proof of Proposition 2) are determined for $d=0.1$. 


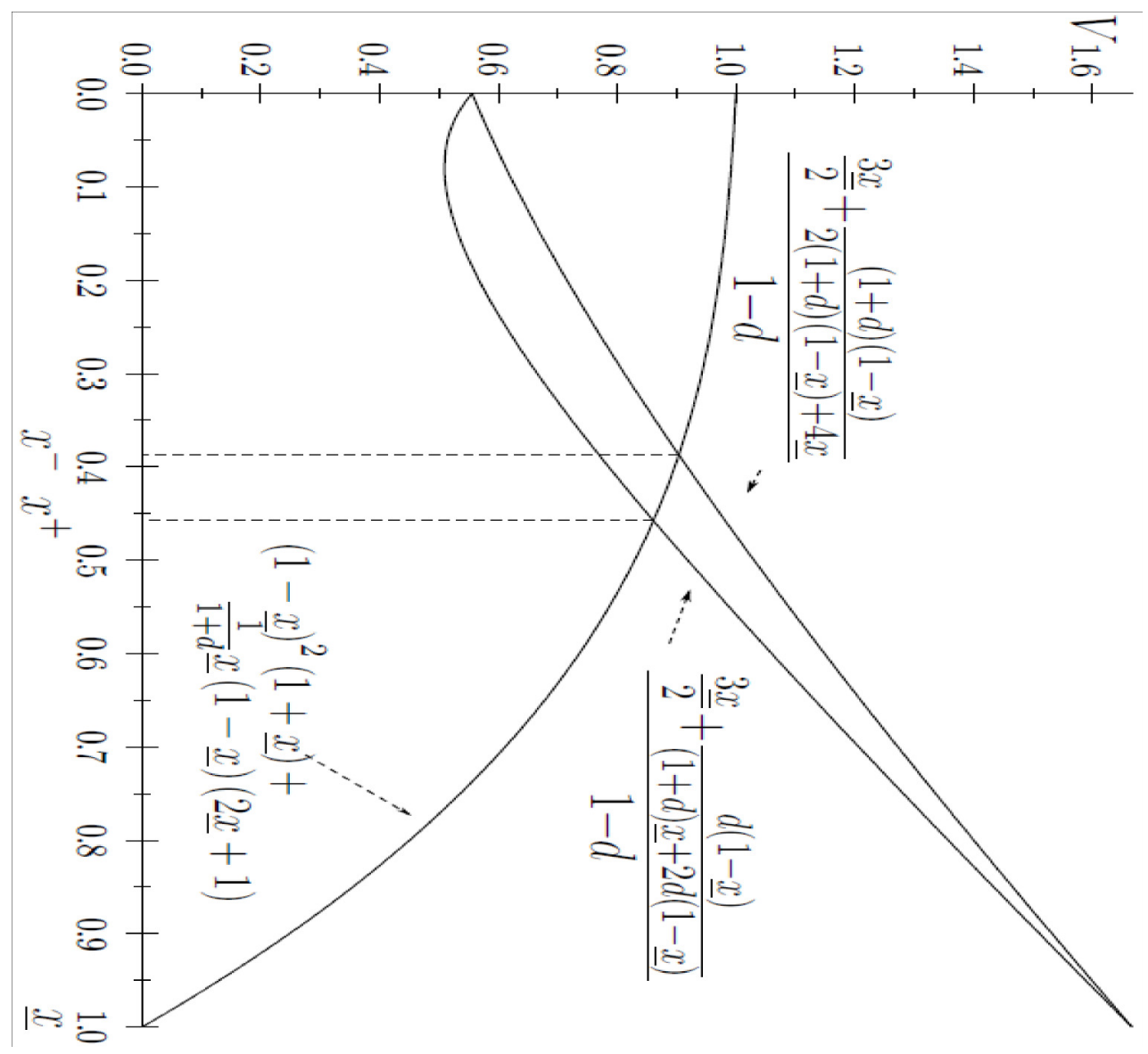

Figure 3 


\section{Appendix B (online)}

This appendix contains two diagrams that complement the derivations in the main text. Figure 4 depicts the sets of all (not necessarily symmetric) equilibrium threshold pairs for $d=0.1$ and $d=0.3$.

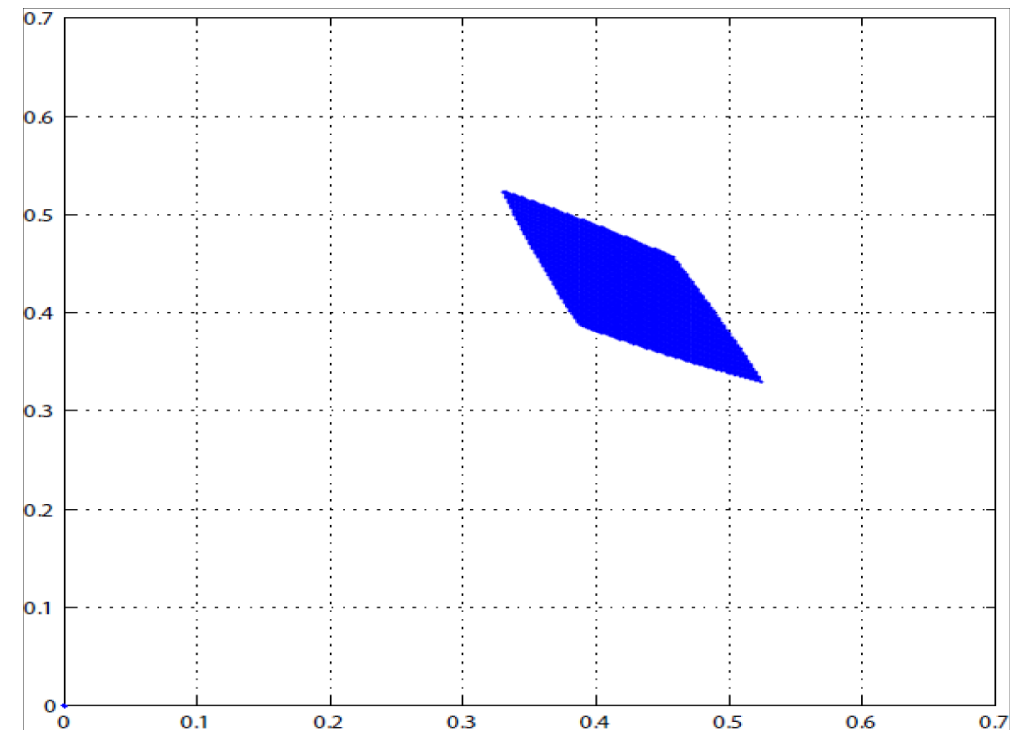

Figure 4(a)

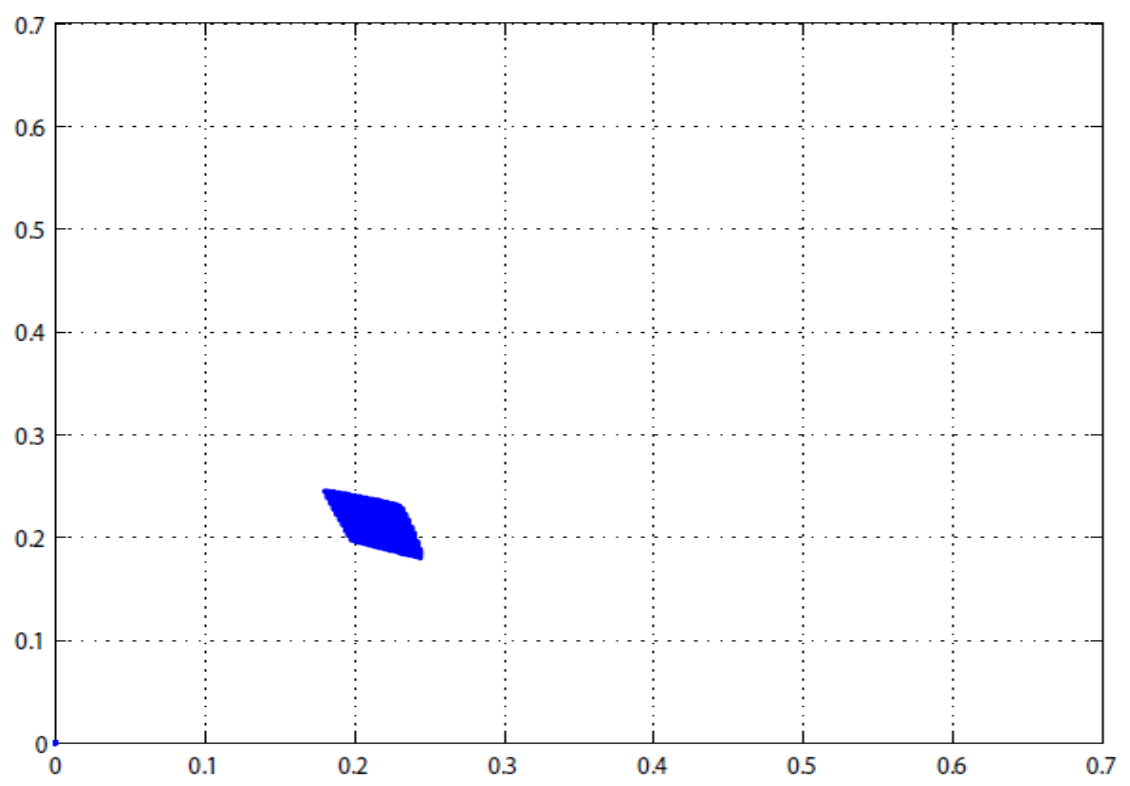

Figure 4(b) 
Figure 5 depicts the graphs of the two functions from the end of Proposition 7

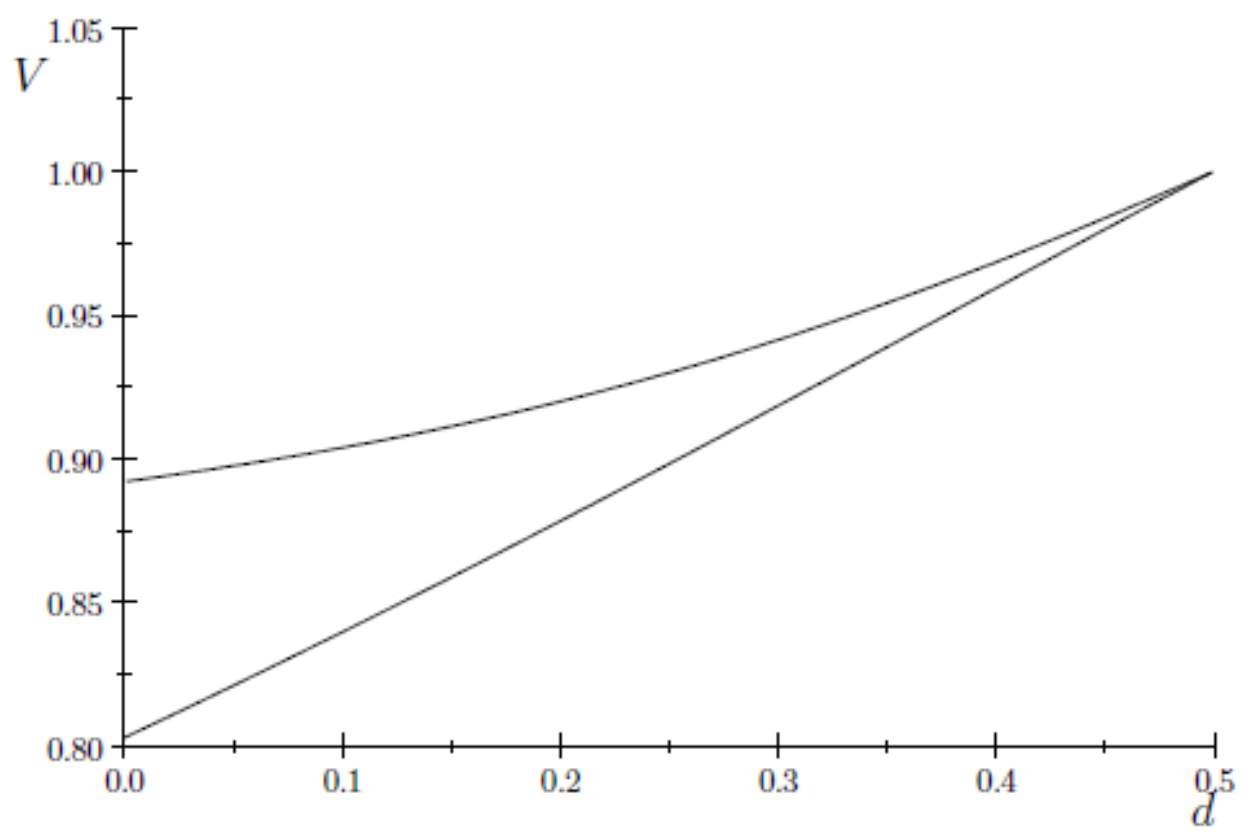

Figure 5 